\title{
COMMENT
}

\section{The EXTEND system for extrauterine support of extremely premature neonates: opportunity and caution}

\author{
Mark R. Mercurio ${ }^{1}$
}

Investigators have recently described successful use of an EXTrauterine Environment for Neonatal Development (EXTEND), essentially an artificial womb, to maintain extremely premature fetal lambs ex utero for up to 4 weeks, supporting normal growth and development. The animals were maintained in a fluid environment (Biobag) while nutrition and gas exchange were supported via umbilical catheters and the use of an external membrane oxygenator. The lambs studied correspond to humans at 23-25 weeks' gestation, which is the target range for proposed clinical trials. This new technology offers the possibility of improving outcomes in a patient population with a high rate of mortality and morbidity. However, if safety and efficacy are adequately demonstrated in the lamb model, there remain important ethical considerations worthy of discussion prior to human trials. Moreover, the authors state there is no intention to use EXTEND on patients below the current limit of viability, but this possibility should nevertheless be considered, and should also be the subject of ethical discussion. Analysis of relevant ethical issues, including patient selection, moral status, rights, obligations, and others should precede use of this promising technology in humans.

Pediatric Research (2018) 84:795-796; https://doi.org/10.1038/s41390-018-0198-2

Investigators at the Children's Hospital of Philadelphia have recently described their successful use of an EXTrauterine Environment for Neonatal Development (EXTEND), essentially an artificial womb, to maintain extremely premature fetal lambs ex utero for up to 4 weeks, supporting normal growth and development. This innovation, and their experimental results, are described in two recent publications ${ }^{1,2}$ and an on-line video. ${ }^{3}$ Essentially, the premature lamb is immersed in fluid within a closed "Biobag," as gas exchange and nutrition are provided via umbilical catheters and an external membrane oxygenator. This remarkable work is as much about innovation and invention as it is about research, building on decades of attempts to create an artificial womb. It is fascinating to learn of the problems they encountered and overcame as they refined their system, and eventually developed a successful prototype. Among the most important innovations was the development of a system that does not require an external pump to circulate blood, but rather relies only on the fetal heart.

The authors note that the premature fetal lambs they studied corresponded developmentally at the start of treatment to the clinical target range of 23-25 weeks' gestation in human infants. One could potentially maintain a human subject on this system for 4 weeks, and then transition to an incubator and mechanical ventilation. Such a successful transition has already been demonstrated in the sheep model. The investigators' stated goal is not to lower the line of viability for premature infants in our Neonatal Intensive Care Units (NICUs), currently at 22 weeks' gestation, but to improve outcomes for those at the edge of viability already receiving care and experiencing high rates of morbidity and mortality.

Neonatologists are painfully aware that our tiniest patients often do not survive despite great effort, and substantial expenditure of resources. Also, among those who survive, many are left with significant disability. The observed high mortality and morbidity are in large part a result of premature transition to the extrauterine environment, notably the transition to gas exchange via the lungs, and the positive pressure ventilation currently employed. This innovation is based upon an effort to postpone that transition when early delivery is unavoidable. Alan Flake, the senior author on the papers, notes in the video that discussion with the Food and Drug Administration is in progress, and human trials could be as close as 1 or 2 years away.

This is not natural. Then again, neither is positive pressure ventilation, nor being kept in an air (rather than fluid) environment at 23 weeks' gestation. For physicians, prioritizing what seems natural should not always be our primary objective. It is quite natural for a preterm baby to die from respiratory failure, for a woman to die in childbirth, or for a young man to die from tuberculosis. A major goal of medicine, that should sometimes supersede supporting what is perceived as natural, is the prevention of such events. The fact that this innovation seems unnatural should not alone rule out its use. Moreover, one could argue that, for a human at 23 weeks, providing a fluid environment and nutrition/oxygenation via the umbilical vessels is in some ways more physiologic (or natural) than current standard care. Still, by the lights of some, there may be something inherently wrong with keeping a fetus alive outside of the womb, or with trying to create a "womb" apart from a mother. That concern deserves fair consideration and discussion. In response, however, one might point out that we are already working hard to keep many of these same patients alive outside of their mothers, at the request of the parents. The EXTEND system might provide a means to essentially do the same thing, with better results.

'Department of Pediatrics and Program for Biomedical Ethics, Yale University School of Medicine, New Haven, CT, USA

Correspondence: Mark R. Mercurio (mark.mercurio@yale.edu)

Received: 20 September 2018 Accepted: 22 September 2018

Published online: 1 October 2018 
There are also other important ethical questions deserving of attention. The prospect of human trials in the relatively near future leads one to ask, if adequate animal data support the safety and efficacy of this new technology, which humans should participate? The authors suggest those born at 23 to 24 or 25 weeks' gestation, given their high rate of mortality, and of lifelong morbidity among survivors, such as pulmonary and neurodevelopmental disability. However, recent data at our own institution and many others show that, using current technology, nearly half of babies born at 23 weeks in hospitals with advanced NICUs now survive, as do about two thirds of those born at 24 weeks. Roughly three out of four born at 25 weeks also now survive. ${ }^{4}$ And, while the rate of morbidity is high, most of these survivors will not be left with severe long-term disability. The chance of survival among those born at 22 completed weeks' gestation is clearly low, but accurate and relevant survival data remain somewhat elusive. One important reason is that infants born at 22 weeks often receive no attempt at resuscitation. Overall survival statistics commonly reported fail to account for this, and can thus be misleading. A report from the National Institutes of Health Neonatal Research Network combining data from 24 academic centers in the United States found that, when active attempts to resuscitate were provided to patients born at 22 completed weeks, survival to discharge was nearly one in four.

This is not to suggest that the long-term disabilities that many survivors experience are insignificant, nor that it would be inappropriate to perform a clinical trial of the EXTEND system. This new technology may potentially provide a much better future for many, but a thorough understanding of current outcomes data is needed to determine which patients should be eligible. If this innovation were to be used, it should be as part of a carefully designed and regulated clinical trial, and, at least at first, should only be attempted in infants who would have a poor prognosis with standard treatment. The short-term and long-term problems that might be experienced by human subjects are not yet known. Truly informed parental permission, discussing potential risks and benefits of standard and experimental treatment regimens, as well as "comfort measures only," would be essential.

The authors make it clear that their goal is to improve outcomes for those currently receiving intensive care, but not to push back the current line of viability. They cite mechanical difficulties due to small size, as well as concerns regarding neurodevelopmental impairment. It is entirely possible that they also emphasize this aspect of their intentions to avoid the controversy that trying to move the limit of viability might bring. Their insistence on this point, in any case, might come as reassurance to those currently providing the very difficult and costly care at the edge of viability. It seems likely that most neonatologists and nurses currently providing the challenging and often heartbreaking care of these patients are not anxious to push the threshold even further back. Nevertheless, at some point one might well ask whether a patient just below our current threshold is better served by being given this treatment, and a chance of survival with an unknown risk of disability, rather than being allowed to die with no intervention. The answer, and the ethical justification for the answer, might not be so clear, but the question deserves consideration. Despite current intentions, the line may well be moved at some point, and there is little point in avoiding the discussion.

A clue to one fundamental ethical aspect of this technology can be found in the words of the authors: "An additional disadvantage... is parental perception of having their fetus in a 'bag.' It is important to consider that the comparator is the extreme premature infant on a ventilator and in an incubator. We feel that parents will be relatively reassured that their fetus is being maintained in a relatively protective and physiologic environment." ${ }^{2}$ The point may be valid, but the wording raises a fascinating question. It seems to suggest that the patient on the ventilator is an infant, and the patient in a Biobag is a fetus. This may not be intended, but it is very telling nonetheless. One can find both words used to refer to subjects on this device. Is it a fetus or an infant? Does it matter?

The words we use both reveal and inform how we think, and, as every pediatrician knows, how parents understand their sick child's situation. Imagine twins, one treated with the standard approach and one with a Biobag. The physician might give parents the news that, "Your twins are both doing fairly well right now. Your infant is on a ventilator, and your fetus is in a Biobag." Words do indeed matter, as the assignation of "fetus" or "infant" may carry with it assumptions about moral status, inherent rights, and obligations. A human in a Biobag, particularly at a very early gestational age, may not fit neatly into our understanding of either a fetus or an infant. Questions of nomenclature, moral status, and rights might at times seem a bit esoteric in clinical research, but with the very real possibility of clinical trials and human use of this technology, these questions must be front and center, right alongside technical and physiological considerations. The conversation should include individuals from the medical profession, members of the scientific community, bioethicists, and others from outside of these professional groups.

The investigators are to be congratulated on an extraordinary development. They are correct that there is tremendous potential for studying pregnancy, and the role of the maternal environment and the placenta. There is also potential to improve outcomes for many in our current patient population, as well as (though not their intention) patients who, at present, have no chance of survival. This is exciting for many reasons, not least that it is new territory. It is for that very reason that thoughtful deliberation regarding ethical considerations is essential. Unlike some medical innovations of the past, it is here suggested that the bioethical analysis and discussion occur well before use in the first human subject.

\section{ADDITIONAL INFORMATION}

Competing interests: The author declares no competing interests.

Publisher's note: Springer Nature remains neutral with regard to jurisdictional claims in published maps and institutional affiliations.

\section{REFERENCES}

1. Partridge, E. A. et al. An EXTrauterine environment for neonatal development: EXTENDING fetal physiology beyond the womb. Semin. Fetal Neonatal Med. 22, 404-409 (2017).

2. Partridge, E. A., et al. An extra-uterine system to physiologically support the extreme premature lamb. Nat. Commun. 8, 15112 (2017).

3. The Children's Hospital of Philadelphia. Recreating the Womb: Q\&A with the researchers. https://www.youtube.com/watch?v=2xpKmyCE97A (accessed 14 August 2018).

4. Patel, R. M. et al. Survival of infants born at periviable gestational ages. Clin. Perinatol. 44, 287-303 (2017).

5. Rysavy, M. A., et al. Between-hospital variation in treatment and outcomes in extremely preterm infants. N. Engl. J. Med. 372, 1801-1811 (2015). 